\title{
Microwave Spectrum of Ethyl Iodid: Internal Rotation Analysis
}

\author{
D. Boucher, A. Dubrulle, and J. Demaison \\ Laboratoire de Spectroscopie Hertzienne, Laboratoire associé au C.N.R.S., no. 249, \\ Université de Lille I
}

Z. Naturforsch. 35a, 442-446 (1980); received February 29, 1980

The rotational spectra of the first excited state of the methyl torsion and the CCI-in plane deformation of $\mathrm{CH}_{3} \mathrm{CH}_{2} \mathrm{I}$ have been studied. A Coriolis interaction between these two modes has been found. Splittings of transitions in the first excited torsional state show that the barrier hindering internal rotation of the methyl group is $3.62 \mathrm{kcal} / \mathrm{mole}$. This value agrees quite well with the value previously reported from Raman studies. It is internally consistent and similar to the other ethyl halides.

It was empirically found [1] that the methyl barrier in substituted ethanes $\mathrm{CH}_{3} \mathrm{CH}_{2} \mathrm{X}$ slightly increases when the electronegativity of the substituent $\mathrm{X}$ decreases. Consequently the barrier in ethyl iodide would be expected to be greater than in ethyl fluoride where $V_{3}=3.30 \mathrm{kcal} / \mathrm{mole}$ [2] and of the same order of magnitude as in ethyl bromide: $V_{3}=3.57 \mathrm{kcal} / \mathrm{mole}$ [3]. From a microwave study it was in fact shown that $V_{3}=3.22 \mathrm{kcal} / \mathrm{mole}$ [4], somewhat lower than expected from the above considerations. On the other hand a Raman investigation in gas phase [5] has given $V_{3}=3.67 \mathrm{kcal} / \mathrm{mole}$, which is considerably higher than the value previously reported from microwave studies but in good agreement with the values found for the other ethyl halides.

To clear up this discrepancy we have reinvestigated the microwave spectrum of $\mathrm{CH}_{3} \mathrm{CH}_{2} \mathrm{I}$. The spectra were recorded in the region from 5 to $80 \mathrm{GHz}$ with a conventional microwave spectrometer employing $25 \mathrm{kHz}$ Stark-modulation. For the high resolution measurements a $8 \mathrm{~m}$-absorption cell was used, the sample pressure was $5 \mathrm{~m}$-Torr and the temperature approximately $-50^{\circ} \mathrm{C}$. The analysis of the ground state spectrum is reported elsewhere [6].

${ }^{\mathrm{a}} R_{01}$ transitions of two excited states of nearly equal intensity were easily identified, both at the low frequency side of the ground state transitions. No lines were found to be split. The ${ }^{b} Q_{1,-1}$ lines were much more difficult to find because they were very far from the ground state lines. The ${ }^{\mathrm{b}} Q_{1,-1}$ lines of one excited state shows a doublet splitting while

Reprint requests to be sent to Dr. J. Demaison, U.E.R. de Physique Fondamentale, Université de Lille I, F-59655 Villeneuve d'ASCQ Cedex, France. the other state has very sharp lines. The split lines probably belong to the first excited torsional state which was calculated at $v_{18}\left(a^{\prime \prime}\right)=259 \mathrm{~cm}^{-1}[7]$ and the other state was most likely the in-plane CCI bend, measured in gas phase as $v_{11}\left(a^{\prime}\right)=258 \mathrm{~cm}^{-1}$ [7]. The relative intensities are in agreement with this vibrational assignment.

The energy difference between these two vibrational levels is only about $1 \mathrm{~cm}^{-1}$. Hence, the rotational spectra of these two states are expected to be perturbed by a Coriolis interaction. Indeed, an attempt to fit these spectra to an appropriate centrifugal distorded Hamiltonian [8] gave a poor fit. On the other hand a fit without the ${ }^{\mathrm{b}} Q_{1,-1}$ lines was satisfactory but the A-rotational constants were very inaccurate. To overcome this difficulty we have used a weighted least squares method giving a weight 100 times lower to the ${ }^{\mathrm{b}} Q_{1,-1}$ lines. In this way the ${ }^{a} R_{0,1}$ lines could be well reproduced (see Tables 1, 2 and 3) and the A-rotational constants were also determined (Table 4). These two constants deviate considerably from the ground state $A$ constant in opposite directions. This indicates the existence of an a-type Coriolis interaction between the two states. The state with negative deviation of $A$ is the lower frequency, it is the CCI in-plane deformation. So the torsion lies a few $\mathrm{cm}^{-1}$ above this state. This is in good agreement with the vibrational assignment of Ref. [7].

The A-E doublet splitting of the excited torsional state can be affected by the Coriolis interaction. A treatment of this perturbation has been given by Dreizler and coll. $[9,10,11]$. But for the case of a high barrier and small asymmetry, it was shown by Laurie [12] that the contribution from Coriolis 


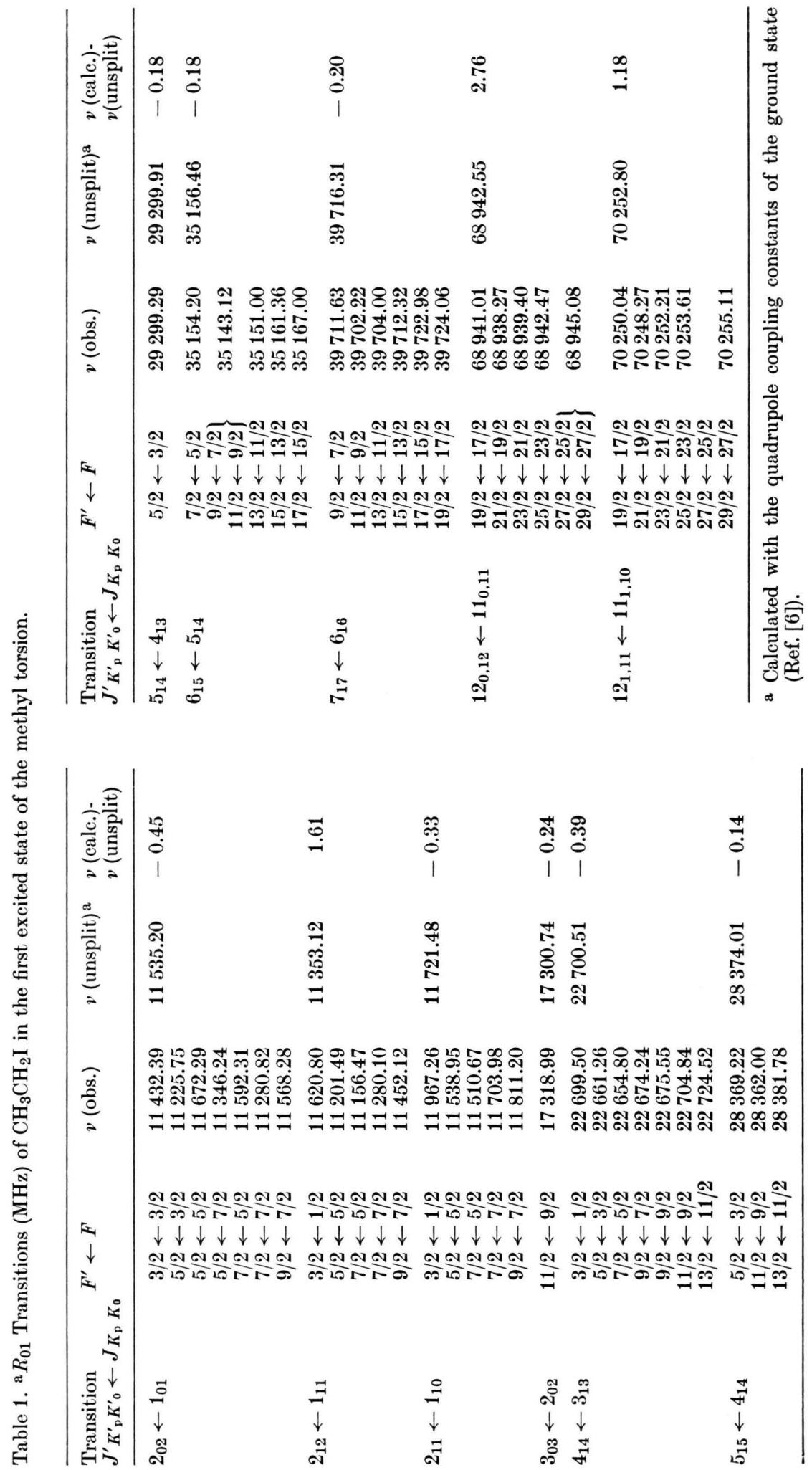


Table 2. ${ }^{\mathrm{b}} Q$ Transitions $(\mathrm{MHz})$ of $\mathrm{CH}_{3} \mathrm{CH}_{2} \mathrm{I}$ in the first excited state of the methyl torsion.

\begin{tabular}{|c|c|c|c|c|c|c|c|}
\hline $\begin{array}{l}\text { Transition } \\
J^{\prime} K_{{ }_{p}}^{\prime} K^{\prime}{ }_{0} \leftarrow J_{K_{\mathrm{p}}} K_{0}\end{array}$ & $F^{\prime} \leftarrow F$ & $\begin{array}{l}\text { Torsional } \\
\text { species }\end{array}$ & $v$ (obs.) & $\begin{array}{l}\text { Internal } \\
\text { rotation } \\
\text { splitting } \\
\text { measured }\end{array}$ & $\begin{array}{l}\text { Internal } \\
\text { rotation } \\
\text { splitting } \\
\text { calculated }\end{array}$ & $\nu$ (unsplit) $^{\mathrm{a}}$ & $\begin{array}{l}v \text { (calc.)- } \\
v \text { (unsplit) }\end{array}$ \\
\hline $8_{17} \leftarrow 8_{08}$ & $\begin{array}{l}11 / 2 \leftarrow 11 / 2 \\
13 / 2 \leftarrow 13 / 2 \\
15 / 2 \leftarrow 15 / 2 \\
17 / 2 \leftarrow 17 / 2 \\
19 / 2 \leftarrow 19 / 2 \\
21 / 2 \leftarrow 21 / 2\end{array}$ & $\begin{array}{l}\text { A } \\
\text { E } \\
\text { A } \\
\text { E } \\
\text { A } \\
\text { E } \\
\text { A } \\
\text { E } \\
\text { A } \\
\text { E } \\
\text { A } \\
\text { E }\end{array}$ & $\begin{array}{l}29943.379 \\
29943.999 \\
29975.333 \\
29975.952 \\
29998.890 \\
29999.511 \\
30006.936 \\
30007.566 \\
29992.941 \\
29993.567 \\
29953.666 \\
29954.284\end{array}$ & $\begin{array}{l}0.621 \\
0.630 \\
0.626 \\
0.618\end{array}$ & 0.638 & 29982.00 & -137.14 \\
\hline $9_{18} \leftarrow 9_{09}$ & $\begin{array}{l}13 / 2 \leftarrow 13 / 2 \\
17 / 2 \leftarrow 17 / 2 \\
19 / 2 \leftarrow 19 / 2 \\
21 / 2 \leftarrow 21 / 2 \\
23 / 2 \leftarrow 23 / 2\end{array}$ & $\begin{array}{l}\text { A } \\
\text { E } \\
\text { A } \\
\text { E } \\
\text { A } \\
\text { E } \\
\text { A } \\
\text { E } \\
\text { A } \\
\text { E }\end{array}$ & $\begin{array}{l}30951.225 \\
30951.900 \\
31005.948 \\
31006.621 \\
31012.992 \\
31013.657 \\
30998.658 \\
30999.332 \\
30960.665 \\
30961.328\end{array}$ & $\begin{array}{l}0.675 \\
0.673 \\
0.667 \\
0.673 \\
0.663\end{array}$ & 0.670 & 30987.496 & -51.39 \\
\hline $10_{1,9} \leftarrow 10_{0,10}$ & $\begin{array}{l}15 / 2 \leftarrow 15 / 2 \\
17 / 2 \leftarrow 17 / 2 \\
19 / 2 \leftarrow 19 / 2 \\
21 / 2 \leftarrow 21 / 2 \\
23 / 2 \leftarrow 23 / 2 \\
25 / 2 \leftarrow 25 / 2\end{array}$ & $\begin{array}{l}\text { A } \\
\text { E } \\
\text { A } \\
\text { E } \\
\text { A } \\
\text { E } \\
\text { A } \\
\text { E } \\
\text { A } \\
\text { E } \\
\text { A } \\
\text { E }\end{array}$ & $\begin{array}{ll}32 & 083.991 \\
32 & 084.707 \\
32 & 116.331 \\
32 & 117.048 \\
32 & 138.932 \\
32 & 139.643 \\
32 & 145.142 \\
32 & 145.853 \\
32 & 130.044 \\
32 & 130.753 \\
32 & 093.317 \\
32 & 094.030\end{array}$ & $\begin{array}{l}0.716 \\
0.717 \\
0.711 \\
0.711 \\
0.709 \\
0.713\end{array}$ & 0.707 & 32120.046 & -37.33 \\
\hline $11_{1,10} \leftarrow 11_{0,11}$ & $\begin{array}{l}17 / 2 \leftarrow 17 / 2 \\
19 / 2 \leftarrow 19 / 2 \\
21 / 2 \leftarrow 21 / 2 \\
25 / 2 \leftarrow 25 / 2\end{array}$ & $\begin{array}{l}\text { A } \\
\text { E } \\
\text { A } \\
\text { E } \\
\text { A } \\
\text { E } \\
\text { A } \\
\text { E }\end{array}$ & $\begin{array}{l}32358.308 \\
32 \quad 359.070 \\
32389.895 \\
32 \\
390.659 \\
33408.994 \\
33409.737 \\
33404.217 \\
33404.989\end{array}$ & $\begin{array}{l}0.762 \\
0.764 \\
0.743 \\
0.772\end{array}$ & 0.750 & 33393.132 & 136.02 \\
\hline
\end{tabular}

a Calculated with the quadrupole coupling constants of the ground state (Ref. [6]) and with $v_{0}=\left(v_{\mathrm{A}}+2 v_{\mathrm{E}}\right) / 3$.

coupling to the A-E splittings is small. This conclusion is furthermore strengthened by the fact that only the torsional excited state shows a splitting due to internal rotation. So the barrier to internal rotation of the methyl group was calculated from the splittings with the conventional principal axis method $[13,14]$, the calculation used second and fourth order perturbation. The imput data for this computation includes, in addition to the observed splittings, the moment of inertia of the methyl top, taken to be $I_{a}=3.18 \mathrm{u}$. $\AA^{2}$, the rotational constants and the angle $\Theta$ (methyl top to a axis) $=48.5^{\circ}$. This latter quantity was obtained from an assumed structure [15]. The derivatives shown in Table 4 
Table 3. Rotational Transitions $(\mathrm{MHz})$ of $\mathrm{CH}_{3} \mathrm{CH}_{2} \mathrm{I}$ in the first excited state of the in plane-CCI deformation.

\begin{tabular}{|c|c|c|c|c|}
\hline $\begin{array}{l}\text { Transition } \\
\boldsymbol{J}^{\prime} \boldsymbol{K}_{\mathrm{p}}^{\prime} \boldsymbol{K}^{\prime}{ }_{0} \leftarrow \boldsymbol{J}_{\boldsymbol{K}_{\mathrm{p}} \boldsymbol{K}}\end{array}$ & $F^{\prime} \leftarrow F$ & $v$ (obs.) & $\nu$ (unsplit) & $\begin{array}{l}v \text { (calc.)- } \\
v \text { (unsplit) }\end{array}$ \\
\hline $2_{02} \leftarrow 1_{01}$ & $\begin{array}{l}3 / 2 \leftarrow 1 / 2 \\
3 / 2 \leftarrow 3 / 2 \\
5 / 2 \leftarrow 3 / 2 \\
5 / 2 \leftarrow 5 / 2 \\
3 / 2 \leftarrow 5 / 2 \\
7 / 2 \leftarrow 5 / 2 \\
5 / 2 \leftarrow 7 / 2 \\
7 / 2 \leftarrow 7 / 2 \\
9 / 2 \leftarrow 7 / 2\end{array}$ & $\begin{array}{l}11609.89 \\
11417.35 \\
11211.84 \\
11656.28 \\
11861.70 \\
11576.60 \\
11346.24 \\
11266.57 \\
11552.74\end{array}$ & 11520.07 & 0.37 \\
\hline $2_{12} \leftarrow 1_{11}$ & $\begin{array}{l}9 / 2 \leftarrow 7 / 2 \\
7 / 2 \leftarrow 5 / 2 \\
7 / 2 \leftarrow 7 / 2\end{array}$ & $\begin{array}{l}11440.77 \\
11146.96 \\
11268.97\end{array}$ & 11341.91 & -0.05 \\
\hline $2_{11} \leftarrow 1_{10}$ & $\begin{array}{l}7 / 2 \leftarrow 5 / 2 \\
5 / 2 \leftarrow 5 / 2 \\
9 / 2 \leftarrow 7 / 2\end{array}$ & $\begin{array}{l}11489.55 \\
11517.81 \\
11699.67\end{array}$ & 11699.63 & 0.25 \\
\hline $3_{03} \leftarrow 2_{02}$ & $11 / 2 \leftarrow 9 / 2$ & 17295.95 & 17277.70 & 0.52 \\
\hline $4_{14} \leftarrow 3_{13}$ & $\begin{array}{c}3 / 2 \leftarrow 1 / 2 \\
5 / 2 \leftarrow 3 / 2 \\
7 / 2 \leftarrow 5 / 2 \\
9 / 2 \leftarrow 7 / 2 \\
11 / 2 \leftarrow 9 / 2 \\
13 / 2 \leftarrow 11 / 2\end{array}$ & $\begin{array}{l}22681.15 \\
22643.15 \\
22636.73 \\
22656.05 \\
22686.56 \\
22706.18\end{array}$ & 22682.29 & 0.40 \\
\hline $5_{15} \leftarrow 4_{14}$ & $\begin{array}{l}11 / 2 \leftarrow 9 / 2 \\
13 / 2 \leftarrow 11 / 2 \\
15 / 2 \leftarrow 13 / 2\end{array}$ & $\begin{array}{l}28339.21 \\
28358.95 \\
28366.26\end{array}$ & 28351.22 & 0.75 \\
\hline $5_{14} \leftarrow 4_{13}$ & $\begin{array}{c}5 / 2 \leftarrow 3 / 2 \\
11 / 2 \leftarrow 9 / 2 \\
13 / 2 \leftarrow 11 / 2 \\
15 / 2 \leftarrow 13 / 2\end{array}$ & $\begin{array}{l}29243.78 \\
29235.64 \\
29248.80 \\
29265.62\end{array}$ & 29244.40 & 0.55 \\
\hline
\end{tabular}

\begin{tabular}{|c|c|c|c|c|}
\hline $\begin{array}{l}\text { Transition } \\
J^{\prime} K_{{ }_{\mathrm{p}}} K^{\prime}{ }_{0} \leftarrow J_{K_{\mathrm{p}}}\end{array}$ & $F^{\prime} \leftarrow F$ & $v$ (obs.) & $v$ (unsplit) & $\begin{array}{l}v \text { (calc.)- } \\
v \text { (unsplit) }\end{array}$ \\
\hline $6_{15} \leftarrow 5_{14}$ & $\begin{aligned} 7 / 2 & \leftarrow 5 / 2 \\
9 / 2 & \leftarrow 7 / 2 \\
11 / 2 & \leftarrow 9 / 2 \\
13 / 2 & \leftarrow 11 / 2 \\
15 / 2 & \leftarrow 13 / 2 \\
17 / 2 & \leftarrow 15 / 2\end{aligned}$ & $\begin{array}{l}35088.19 \\
35076.85 \\
35084.80 \\
35095.22 \\
35100.59\end{array}$ & 35090.25 & 0.54 \\
\hline $7_{17} \leftarrow 6_{16}$ & $\begin{aligned} 9 / 2 & \leftarrow 7 / 2 \\
11 / 2 & \leftarrow 9 / 2 \\
13 / 2 & \leftarrow 11 / 2 \\
15 / 2 & \leftarrow 13 / 2 \\
17 / 2 & \leftarrow 15 / 2 \\
19 / 2 & \leftarrow 17 / 2\end{aligned}$ & $\begin{array}{l}39680.26 \\
39669.90 \\
39671.62 \\
39680.90 \\
39690.95 \\
39692.04\end{array}$ & 39684.38 & 0.73 \\
\hline $9_{18} \leftarrow 9_{09}$ & $23 / 2 \leftarrow 23 / 2$ & 30370.11 & 30396.81 & 129.48 \\
\hline $10_{19} \leftarrow 10_{0,10}$ & $\begin{array}{l}15 / 2 \leftarrow 15 / 2 \\
17 / 2 \leftarrow 17 / 2 \\
19 / 2 \leftarrow 19 / 2 \\
21 / 2 \leftarrow 21 / 2 \\
23 / 2 \leftarrow 23 / 2 \\
25 / 2 \leftarrow 25 / 2\end{array}$ & $\begin{array}{ll}31 & 279.67 \\
31 & 311.45 \\
31 & 334.77 \\
31 & 342.73 \\
31 & 328.88 \\
31 & 289.85\end{array}$ & 31316.17 & 42.65 \\
\hline $11_{1,10} \leftarrow 11_{0,11}$ & $\begin{array}{l}17 / 2 \leftarrow 17 / 2 \\
19 / 2 \leftarrow 19 / 2 \\
21 / 2 \leftarrow 21 / 2 \\
23 / 2 \leftarrow 23 / 2 \\
25 / 2 \leftarrow 25 / 2 \\
27 / 2 \leftarrow 27 / 2\end{array}$ & $\begin{array}{ll}32 & 323.97 \\
32 & 355.51 \\
32 & 378.01 \\
32 & 384.97 \\
32 & 370.93 \\
32 & 333.20\end{array}$ & 32359.44 & -45.88 \\
\hline $12_{0,12} \leftarrow 11_{0,11}$ & $\left.\begin{array}{l}19 / 2 \leftarrow 17 / 2 \\
21 / 2 \leftarrow 19 / 2 \\
23 / 2 \leftarrow 21 / 2 \\
25 / 2 \leftarrow 23 / 2 \\
27 / 2 \leftarrow 25 / 2 \\
29 / 2 \leftarrow 27 / 2\end{array}\right\}$ & $\begin{array}{l}68853.58 \\
68850.78 \\
68851.94 \\
68855.01 \\
68857.71\end{array}$ & 68855.07 & -1.26 \\
\hline $13_{1,12} \leftarrow 12_{1,11}$ & $31 / 2 \leftarrow 29 / 2$ & 75950.05 & 75948.04 & -0.31 \\
\hline
\end{tabular}


Table 4. Rotational constants of $\mathrm{CH}_{3} \mathrm{CH}_{2} \mathrm{I}^{\text {a }}$.

\begin{tabular}{lrll}
\hline & Ground state $^{\mathrm{b}}$ & $\mathrm{CH}_{3}$-torsion & CCI-bend $^{\mathrm{c}}$ \\
\hline$A$ & $29116.321(8)$ & $29515(41)$ & $28596(40)$ \\
$B$ & $2979.564(1)$ & $2976.74(7)$ & $2969.52(4)$ \\
$C$ & $2796.452(1)$ & $2791.59(6)$ & $2790.81(5)$ \\
$\alpha^{A}$ & & -399 & +520 \\
$\alpha^{B}$ & & 2.82 & 10.04 \\
$\alpha^{C}$ & & 4.86 & 5.64 \\
\hline
\end{tabular}

a All values in MHz. The uncertainties shown in parentheses are in units of the last digit and are standard errors.

b From Ref. [6].

c The ground state centrifugal distortion constants of Ref. [6] were used in the fit.

indicate the dependence of $V_{3}$ on the two fixed parameters $I_{a}$ and $\Theta$ and on the rotational constant $A$, which is not accurately determined. The error of

[1] I. Botskor, Habilitations thesis, Ulm 1979.

[2] D. R. Herschbach, J. Chem. Phys. 25, 358 (1956).

[3] D. R. Lide, J. Chem. Phys. 30, 37 (1959).

[4] T. Kasuya, J. Phys. Soc. Japan 15, 1273 (1960).

[5] J. R. Durig, W. E. Bucy, L. A. Carreira, and C. J. Wurrey, J. Chem. Phys. 60, 1754 (1974).

[6] D. Boucher, A. Dubrulle, and J. Demaison, J. Mol. Spectrose; in press.

[7] J. R. Durig, J. W. Thompson, V. Thyagesan, and J. D. Witt, J. Mol. Struct. 24, 41 (1975).

[8] B. P. van Eijck, J. Mol. Spectr. 53, 246 (1974).
Table 5. Internal rotation parameters of $\mathrm{CH}_{3} \mathrm{CH}_{2} \mathrm{I}$.

$$
\begin{aligned}
& s=96.46(12)^{\mathrm{a}} \quad V_{3}=3623 \pm 150 \mathrm{cal} / \mathrm{mole}^{\mathrm{b}} \\
& \text { [with } I_{\alpha}=3.18 \mathrm{u} . \AA^{2} \text { and } \Varangle(i, a)=48.5^{\circ} \text { assumed] } \\
& \partial V_{3} / \partial \theta=-22 \mathrm{cal} / \mathrm{mole} \mathrm{deg} ; \\
& \partial V_{3} / \partial I_{\mathrm{a}}=-872 \mathrm{cal} / \mathrm{mole} \mathrm{u.} \AA^{2} ; \\
& \partial V_{3} / \partial A=3.32 \cdot 10^{-2} \mathrm{cal} / \mathrm{mole} \mathrm{MHz}
\end{aligned}
$$

a One standard error in units of the last digit.

b Allowance of uncertainty of $I_{\alpha}, \Varangle(i, a)$ and $A$ and for the Coriolis effect included.

$V_{3}$ in Table 5 makes an estimated allowance for the uncertainty of $I_{a}, \Theta$ and $A$ and for the possible Coriolis effect.

[9] H. Dreizler, Z. Naturforsch. 23a, 1077 (1968).

[10] H. Mäder, U. Andresen, and H. Dreizler, Z. Naturforsch. 28a, 1163 (1973).

[11] H. Mäder, H. M. Heise, and H. Dreizler, Z. Naturforsch. 29a, 164 (1974).

[12] V. W. Laurie, J. Chem. Phys. 31, 1500 (1959).

[13] C. C. Lin and J. D. Swalen, Rev. Mod. Physics 31, 841 (1959).

[14] H. Dreizler, Fortschr. Chem. Forsch. 10, 59 (1968).

[15] T. Kasuya and T. Oka, J. Phys. Soc. Japan 15, 296 (1960). 